\title{
Localization of type II and type III radio burst sources using multi-spacecraft observations
}

\author{
G. Thejappa ${ }^{1}$ and R. J. MacDowall ${ }^{2}$ \\ ${ }^{1}$ Department of Astronomy, University of Maryland, \\ College Park, MD 20742, USA \\ email: thejappa@astro.umd.edu \\ ${ }^{2}$ NASA, Goddard Space Flight Center \\ Greenbelt, MD 20771 USA \\ email: Robert.MacDowall@nasa.gov
}

\begin{abstract}
A method for the localization of the radio burst sources associated with the flare accelerated electron beams and coronal mass ejection (CME) shocks is presented. This method involves the computations of the ray trajectories, time delays, and optical depths in the refracting solar atmosphere. The coordinates of the radiating source can be obtained by comparing the time delays and intensity ratios of the bursts observed by widely separated spacecraft with the computed group delays and intensity ratios at exit points of the rays from the solar atmosphere. This method is applied to a type III radio burst observed by the STEREO spacecraft.
\end{abstract}

Keywords. Sun, Radio Bursts

\section{Introduction}

The most intense radio emissions from the the solar corona and interplanetary medium are the type III and type II radio bursts, probably excited by the plasma mechanism at the fundamental and second harmonic of the electron plasma frequency, $f_{p e}(k H z)=$ $9 \sqrt{N_{e}\left(\mathrm{~cm}^{-3}\right)}$, where $N_{e}$ is the electron density. These bursts are characterized respectively by the fast and slow frequency drifts in the dynamic spectrum. For a given density model, the plasma emission mechanism allows us to convert the observed frequency drifts into the speeds of the outward propagating exciting agencies. For example, the frequency drifts of type III and type II radio bursts yield speeds typical of the flare accelerated electron beams and coronal mass ejection (CME) driven shocks, respectively. The actual association between type III and type II bursts and flare electron beams and CME shocks has been well-established by in situ observations. At kilometric and hectometric wavelengths, the direction finding and triangulation techniques (Reiner et al. 1998; Hoang et al. 1998) as well as the time difference of the arrival (TDOA) technique (Weber et al. 1977) are usually used to obtain the positions of the source observed by two or more spacecraft. Even though the triangulation technique requires the 2 rays from the 2 spacecraft to intersect at a point, such intersection usually does not occur due to refraction and scattering. For example, Hoang et al. (1998) have determined the position of a type II burst source using triangulation technique, and found it to be off by several AU from the actual source location due to refraction and scattering. As far as the TDOA technique is concerned, the underlying assumptions are that (1) the effects of propagation on the radio emissions are negligible, and (2) the loci of points that have a fixed difference of distance between two points is a hyperbola (or a hyperboloid) of revolution. Under such circumstances, one can fix the position of the source to a point on such a 
hyperbola either by assuming that these bursts are excited at the $f_{p e}$ or $2 f_{p e}$, or by using observations from an additional spacecraft. However, the radio emissions as they propagate through the inhomogeneous plasma experience time delays due to refraction and scattering. For example, Steinberg et al. (1984) have measured the arrival times of the type III radio bursts at the ISEE- 3 and Voyager 1 and 2 spacecraft and have found that the radio signals are anomalously delayed up to 500 seconds equivalent to $1 \mathrm{AU}$ of excess path length or even more, when the bursts were detected at two spacecraft. Therefore, unless the measured directions and time delays are corrected for propagation effects, the results obtained by these techniques are very unreliable.

The purpose of this paper is to describe a new method, which can be used to localize the radio burst sources observed by a pair of widely separated spacecraft. This method is based on the time delays and attenuations suffered by the radio emissions due to propagation effects, especially refraction. In section 2 , we describe the density and temperature models used in this study, in section 3, we present the ray tracing equations and algorithm to compute the ray trajectories, time delays and optical depths, and in section 4, we apply this method to STEREO observations of a type III radio burst.

\section{Density and Temperature Models}

For the electron number density of the corona and interplanetary medium, we use the empirical formula derived by Guhathakurta, Holzer \& MacQueen (1996) based on the Skylab observations obtained during the declining phase of solar cycle 20 (1973-1976)

$$
N_{e}\left(r, \theta_{m g}\right)=N_{p}(r)+\left[N_{c s}(r)-N_{p}(r)\right] e^{-\theta_{m g}^{2} / w^{2}(r)} \mathrm{cm}^{-3} .
$$

Here $r$ is the radial distance in units of $R_{\odot}$, and $\theta_{m g}=\sin ^{-1}\left[-\cos \theta \sin \alpha \sin \left(\phi-\phi_{0}\right)+\right.$ $\sin \theta \cos \alpha]$ is the heliomagnetic latitude of a point from the current sheet, $\theta$ and $\phi$ are the heliographic latitude and longitudes, respectively, $\alpha \simeq 15 \mathrm{deg}$ is the tilt angle between dipole axis and the rotation axis, and $\phi_{0} \simeq 0$ is the angle between the heliomagnetic and heliographic equators. The electron densities at the current sheet $N_{c s}(r)$ and at the poles $N_{p}(r)$ are given by $N_{e}(r)=\Sigma_{i=1}^{3} c_{i} r^{-d_{i}}$, where $c_{1}, c_{2}$ and $c_{3}$ are $1.07,19.94$, and 22.10 for the current sheet, and $0.14,8.02$, and 8.12 for the pole, in units of $10^{7}$, and $d_{1}, d_{2}$ and $d_{3}$ are $2.8,8.45$, and 16.87 , respectively. The half-angular width of the current sheet is $w(r)=\Sigma \gamma_{i} r^{-\delta_{i}}$, where $\gamma_{1}, \gamma_{2}$, and $\gamma_{3}$ are 16.3, 10.0, and 43.20 degrees, and, $\delta_{1}, \delta_{2}$, and $\delta_{3}$ are $0.5,7.31$, and 7.52 , respectively. The expression for collision frequency can be written as $\nu=4.36 N_{e} T_{e}^{-3 / 2}\left[10.8+\ln \left(T_{e}^{3 / 2} / f\right)\right]$, where $T_{e}$ is the electron temperature. In this study, we take $T_{e}=1.5 \times 10^{5} \mathrm{~K}$. The refractive index can be written as $\mu^{2}(r)=1-\frac{f_{p e}^{2}(r)}{f^{2}}$.

\section{Ray Tracing Calculations}

We use the Cartesian coordinate system with origin at the center of the Sun, and x-axis directed along the line of sight. Haselgrove (1963) has shown that the ray tracing can be performed by using a set of 6 first-order differential equations

$$
\begin{aligned}
& \frac{d \vec{R}}{d p}=\vec{T} \\
& \frac{d \vec{T}}{d p}=D(\vec{R})=\frac{1}{2} \frac{\partial \mu^{2}}{\partial \vec{R}} .
\end{aligned}
$$


Here

$$
\vec{R} \equiv\left(\begin{array}{c}
x \\
y \\
z
\end{array}\right) \quad \text { and } \quad \vec{T} \equiv\left(\begin{array}{c}
T_{x} \\
T_{y} \\
T_{z}
\end{array}\right)
$$

are the position and direction vectors, respectively with $T_{x}^{2}+T_{y}^{2}+T_{z}^{2}=\mu$, and $p$ is the independent variable related to the path length as $d p=\frac{d s}{\mu}$. Using equation (2.1), we can write

$$
D(\vec{R}) \equiv \frac{1}{2}\left(\begin{array}{c}
\frac{\partial \mu^{2}}{\partial x} \\
\frac{\partial \mu^{2}}{\partial y} \\
\frac{\partial \mu^{2}}{\partial z}
\end{array}\right)=\frac{8.90 \times 10^{12}}{f^{2}} \frac{1}{r^{4}} N_{e}\left(r, \theta_{m g}\right) \vec{R} .
$$

The 3rd order Runge-Kutta algorithm, which is used to integrate these ray tracing equations can be written in the vectorial form as

$$
\begin{aligned}
R_{n+1} & =R_{n}+\Delta \tau\left[T_{n}+\frac{1}{6}(A+2 B)\right], \\
T_{n+1} & =T_{n}+\frac{1}{6}(A+4 B+C), \\
A & =\Delta \tau D\left(R_{n}\right), \\
B & =\Delta \tau D\left(R_{n}+\frac{\Delta \tau}{2} T_{n}+\frac{1}{8} \Delta \tau A\right), \\
C & =\Delta \tau D\left(R_{n}+\Delta \tau T_{n}+\frac{1}{2} \Delta \tau B\right), \\
D(R) & =\frac{1}{2} \frac{\partial \mu^{2}}{\partial R} .
\end{aligned}
$$

Using this algorithm, one can trace the rays through any medium, i.e., starting from a known point $\left(\overrightarrow{R_{0}}, \overrightarrow{T_{0}}\right)$, one can generate successively $\left(\overrightarrow{R_{1}}, \overrightarrow{T_{1}}\right),\left(\overrightarrow{R_{2}}, \overrightarrow{T_{2}}\right) \ldots \ldots\left(\overrightarrow{R_{n}}, \overrightarrow{T_{n}}\right)$. For example, Thejappa, MacDowall \& Kaiser (2007) and Thejappa \& MacDowall (2008) have successfully applied this algorithm to study the visibility of type III radio bursts and quiet sun radio emissions, respectively. In Fig. 1, we present the computed ray trajectories at $625 \mathrm{kHz}$, where the smaller circle corresponds to the Sun, and the bigger circle corresponds to the $2 f_{\text {pe }}$ plasma level. The optical depth $\tau$ and the transit time $\Delta t$

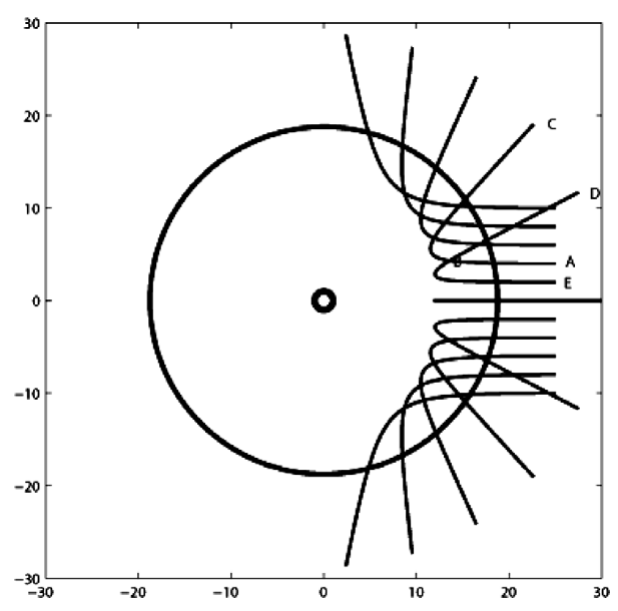

Figure 1. Ray trajectories at $625 \mathrm{kHz}$. 

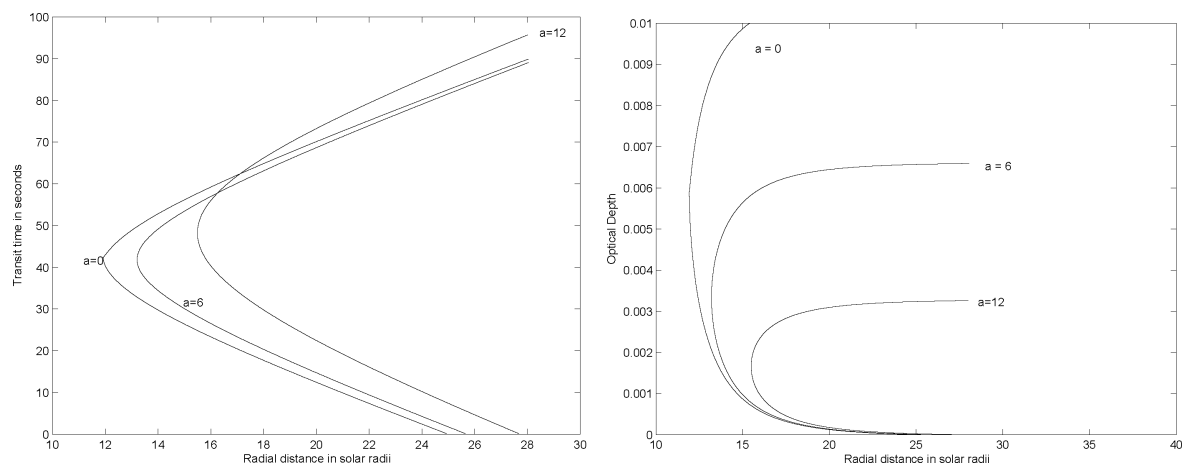

Figure 2. Computed time delays and optical depths.

are computed at each step $\Delta S$ as

$$
\begin{aligned}
\tau_{i+1} & =\tau_{i}+2.32 \frac{f_{p e}^{2}}{f^{2}} \frac{\nu \Delta S}{\mu_{i}} \\
\Delta t_{i+1} & =\Delta t_{i}+2.32 \frac{\Delta S}{\mu_{i}} .
\end{aligned}
$$

The factor 2.32 is the solar radius $\left(R_{\odot}\right)$ divided by the velocity of light $(c)$. In these two equations, instead of phase velocity $c / \mu$, the group velocity $(c \mu)$ is used to relate $\Delta t$ to $\Delta S$. In Fig. 2, we present the computed time delays and optical depths against the radial distance, $r$ for various values of the impact distances, $a$ (the distance of the asymptote of the ray from the parallel line through the sun's center).

\section{Application}

The STEREO mission (Kaiser 2005) consists of two identical spacecraft- one ahead of Earth in its orbit (Stereo 'A'), the other trailing behind (Stereo 'B'). Each spacecraft is equipped with identical instrumentation. For example, the radio receivers (HFR and LFRhi) of the STEREO/Waves experiment (Bougeret et al. 2007) measure the radio wave intensities in the frequency from $40 \mathrm{kHz}$ to $16 \mathrm{MHz}$, corresponding to source distances of about $1 \mathrm{AU}$ to $1 R_{\odot}$. This experiment is mainly designed to study type II and type III radio bursts. In Fig. 3, we present a typical type II radio burst and a typical type III radio burst detected by both the spacecraft on December 31, 2007. The spacecraft were separated from each other by $\sim 42$ degrees. The top part of the dynamic spectrum
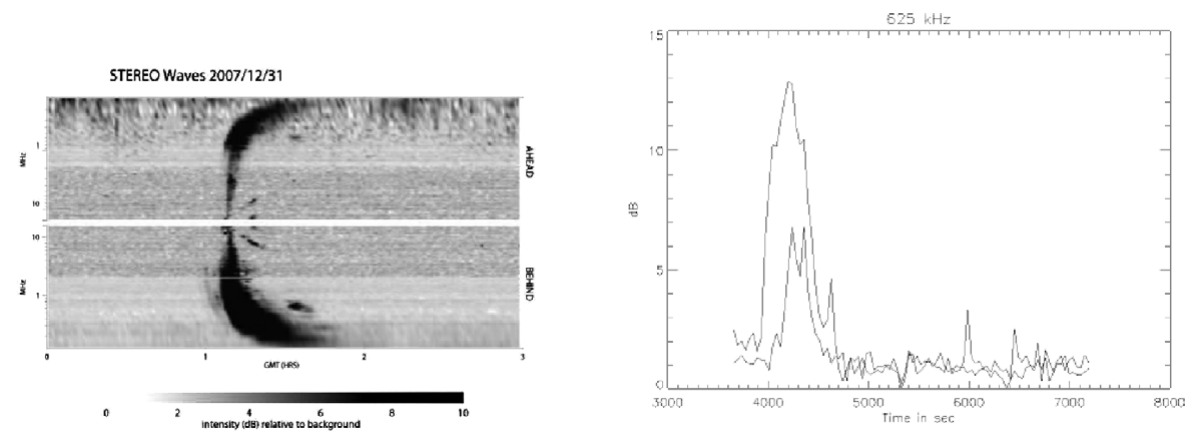

Figure 3. The dynamic Spectrum and the time profile of the type III radio burst observed simultaneously by the two spacecraft of the STEREO mission. 
extending from 1 to $16 \mathrm{kHz}$ is obtained by STEREO 'A', and the bottom part extending from $16 \mathrm{MHz}$ to $1 \mathrm{MHz}$ is obtained by STEREO 'B'. The dynamic spectra from both spacecraft clearly show that type III burst extends all the way from $16 \mathrm{MHz}$ to $1 \mathrm{MHz}$ and the slowly drifting type II radio burst contains both fundamental and harmonic bands. In this dynamic spectrum, the LFR frequencies are not shown. Fig. 3 also shows the time profiles of the type III burst (top and bottom profiles correspond to STEREO 'A' and 'B', respectively). From these time profiles, it is clear that the type III emission is delayed almost by $\sim 72$ seconds at ' $\mathrm{B}$ ' in comparison with that of ' $\mathrm{A}$ '. Here we note that the time delay is estimated using the start time of these bursts since the peak of the emission from ' $\mathrm{B}$ ' is difficult to establish. Moreover, the burst at ' $\mathrm{B}$ ' is much weaker than that of ' $\mathrm{A}$ ' by a factor of $\sim 2$. The computed ray trajectories, time delays and optical depths in the previous section provide important clues to understanding these observed time delays and intensity ratios. A possible interpretation is that (1) the type III burst is generated by a localized disturbance in the solar atmosphere, and (2) the radiation from the disturbance reaches the widely separated spacecraft by two different paths, namely "direct" path and "echo" path. The "echo" is delayed and attenuated in comparison with the "direct" signal because it has to travel a larger distance with considerably slower group velocity to reach the receiver. For example, these anamolies can be understood using the computed trajectories (Fig. 1). Let us assume that the type III emission is generated by the disturbance located at $\mathrm{B}$. This radiation can reach 'A' by the path BA, and the 'B' by the path BC approximately separated by $40 \mathrm{deg}$. As seen from these trajectories the "echo" signal which follows the path BC will be more attenuated and will arrive at C later than "direct"' signal along BA. These time delays and attenuations can be determined from Figure 2. The practical aspect of these anamolies is that by comparing the observed time delays and attenuations with observed quantities, we can find the coordinates of the source (localization). For example, the three dimensional localization of the radio source at one frequency fixes the instantaneous location of the electron beam in the case of a type III burst, and the location of the CME shock in the case of a type II radio burst. By repeating the source localizations at several frequencies, the trajectories of the electron beams and CME shocks can be mapped through interplanetary space. By successfully tracking the radio sources, one can predict the arrival of the electron beams and CME shocks at Earth which is essential for space weather predictions. The detailed procedure and algorithm of localization and tracking of radio sources using the present method will be presented in a separate paper.

Support for T. G. was provided by the Living With a Star Targeted Research and Technology program through NASA Grant 07-LWSTRT-0120. We thank M. L. Kaiser for providing the data and R. A. Hess for his help with data.

\section{References}

Bougeret et al. 2008, Space Sci. Rev., 136, 487.

Guhathakurta, M., Holzer, T. E., \& MacQueen, R. M. 1996, ApJ, 458, 817

Haselgrove, J. 1963, J. Atmos. Terr. Phys., 25, 397

Hoang, S., Maksimovic, M., Bougeret, J.-L., Reiner, M. J., \& Kaiser, M. L. 1998, GRL, 25, 2497

Kaiser, M. L., 2005, Advances in Space Res., 36, 1483

Reiner, M. J., Fainberg, J., Kaiser, M. L., \& Stone, R. G. 1998, JGR, 103, 1925.

Steinberg, J.-L., Dulk, G. A., Hoang, S., Lecacheux, A., \& Aubier, M. G. 1984, A\&A, 140, 39

Thejappa, G., MacDowall, R. J., \& Kaiser, M. L. 2007, ApJ, 671, 894

Thejappa, G. \& MacDowall, R. J. 2008, ApJ, 676, 1338

Weber, R. R., Fitzenreiter, R. J., Novaco, J. C., \& Fainberg, J. 1977, Solar Phys., 54, 431 


\section{Discussion}

Anonymous: Can you measure a real angular size of the cone type III emission propagates in?

ThEJAPPA: It is known that type III emission should be beamed if it is excited by plasma mechanism. However, they are visible to widely separated spacecraft. To desire the intrinsic beam pattern, we have to correct the observed visibility for the propagation effects by doing statistical ray tracing. 